\title{
Medida da Translucência Nucal no Rastreamento de Anomalias Cromossômicas
}

\author{
Nuchal Translucency Measurement in the Screening of Chromosomal Abnormalities \\ Carlos Geraldo Viana Murta, Luiz Cláudio França
}

\begin{abstract}
RESUMO
Objetivo: estudar o método para medida da translucência nucal (TN) e a sua aplicabilidade no rastreamento de anomalias cromossômicas entre a $10^{a}$ e a $14^{a}$ semanas de gestação. Métodos: foram estudados 1152 fetos consecutivamente. Em 124 casos realizou-se estudo citogenético no material obtido por meio da biópsia de vilosidade coriônica ou da amniocentese e em 1028 o resultado teve como base o fenótipo do recém-nascido. Todos os fetos foram submetidos, além da ultra-sonografia de rotina, à medida da TN. Na análise estatistica foram utilizados os testes paramétricot de Student e análise de variância. Posteriormente, calcularamse: sensibilidade, especificidade, valor preditivo positivo e negativo, probabilidade de falsopositivo e razões de probabilidades.

Resultados: foram diagnosticados 23 casos de anomalias cromossômicas na população estudada. Dentre esses, a medida da TN foi maior que o percentil 95 em 16 (sensibilidade de 69,5\%). No grupo de fetos normais (1 129 casos) a medida da TN foi maior que o percentil 95 em 41 avaliações (especificidade de 96,3\%, valor preditivo positivo e negativo de 28,0\% e 99,3\%, respectivamente, taxa de falso-positvo de 3,7\% e razão de probabilidade de 19,1). Conclusão: nossos resultados sugerem que a presença de anomalias cromossômicas pode ser fortemente suspeitada quando existir aumento da TN. Depreende-se que basta a análise quantitativa da medida da TN para categorizar o risco de anomalias cromossômicas no primeiro trimestre. Portanto, ainda que seja necessário treinamento e habilidade do operador, trata-se de método de aplicabilidade clinica.
\end{abstract}

PALAVRAS-CHAVE: Translucência nucal. Anomalias cromossômicas. Diagnóstico pré-natal.

\section{Introdução}

Alguns estudos recentes analisaram o rastreamento das aneuploidias no primeiro trimestre da gestação com base no emprego da medida da translucência nucal (TN) em associação com a idade materna ou com marcadores séricos ${ }^{1-4}$. A TN configura-se como imagem ultra-sonográfica hipoecogênica, acúmulo de líquido na região posterior do pescoço, que ocorre com mais exuberância entre a $10^{\mathrm{a}}$ e a $14^{\mathrm{a}}$ semanas de gestação ${ }^{1}$.

Desde o início dos anos 90, diversos estudos com pequenas casuísticas e com populações de alto

Universidade Federal do Espírito Santo e Vitória MedifetUS Correspondência:

Carlos G. V. Murta

Rua das Palmeiras, 795/409 - Santa Lúcia

29045-205 - Vitória - ES

Tel.: (27) 3225-1343, (27) 3227-2543 - Fax: (27) 315-4472

e-mail: cgvmurta@zaz.com.br risco demonstraram associação entre o aumento da espessura da TN, entre a $10^{\mathrm{a}}$ e a $14^{\mathrm{a}}$ semanas, e a presença de anomalias cromossômicas ${ }^{1,2,5}$.

Inicialmente as taxas de detecção da sindrome de Down por meio da avaliação ultrasonográfica apresentavam grande variação entre os diversos estudos publicados, variando de $29 \%$ a $75 \%$, usando como critério de anormalidade um ponto de corte fixo ${ }^{5-9}$. Essa ampla variação deve-se a critérios de seleção não adequados e não comparáveis, destacando-se o fato de muitos trabalhos terem empregado o ponto de corte fixo em 3,0 $\mathrm{mm}$ como critério discriminatório ${ }^{1}$. A diversidade nos resultados também reflete as diferenças na distribuição da idade materna e na definição da medida da TN minima anormal.

Destaca-se o estudo de Pandya et al. ${ }^{2}$ que, examinando 20.804 grávidas, observaram que a TN estava acima do percentil 95 em $77 \%$ dos fetos 
com trissomia do cromossomo 21 e em $80 \%$ das outras anomalias cromossômicas, com taxa de falso-positivo de 4,9\%.

Em um estudo multicêntrico envolvendo cerca de 100.000 gestações se demonstrou que, em $72 \%$ do fetos com trissomia 21 , a medida da TN encontrava-se acima do $95^{\circ}$ percentil da curva de normalidade e o rastreamento empregando a idade materna associada à TN identificou $77 \%$ dos fetos anormais, com resultado falso-positivo de $5 \%{ }^{3}$.

Os mecanismos fisiopatológicos que explicam esse marcador ultra-sonográfico transitório ainda não estão bem estabelecidos. Alguns eventos que ocorrem nessa época da prenhez poderiam eventualmente esclarecer o acúmulo transitório de líquido na região da nuca do feto, que é atribuído a alterações da drenagem linfática fetal e/ou, particularmente, a distúrbio hemodinâmico do feto (disfunção cardíaca) ${ }^{10,11}$.

A insuficiência cardiaca precoce e transitória tem sido apontada como a base primordial do mecanismo responsável pela associação TN aumentada e aneuploidias ${ }^{10,11}$. A confirmação bioquímica de semelhante hipótese advém do aumento do fator natriurético atrial no tecido cardiaco de fetos com trissomias ${ }^{12}$. Nesse particular, o aumento da TN faz supor a possibilidade da existência de cardiopatia, achado incontestado na síndrome de Down. Estudos anatomopatológicos demonstraram, no primeiro trimestre da gestação, que $65 \%$ de fetos portadores dessa anomalia apresentavam defeitos septais ${ }^{13}$ e $49 \%$ exibiram estreitamento do istmo aórtico ${ }^{14}$.

O objetivo desta pesquisa foi estudar a validade do método de medida ultra-sonográfica da TN no rastreamento de anomalias cromossômicas entre a $10^{\mathrm{a}}$ e a $14^{\mathrm{a}}$ semanas de gestação.

\section{Pacientes e Métodos}

O presente estudo compreende uma investigação transversal e prospectiva de 1283 gestações que foram incluídas no estudo consecutivamente, no período de setembro de 1997 a junho de 2001. Trata-se de estudo de validação de teste diagnóstico. Inicialmente foram excluídos desse estudo os abortamentos (9 casos) e 122 casos em que não foi possivel ter o cariótipo ou fenótipo dos recém-nascidos, tornando-se elegivel um total de 1152 fetos.

As pacientes foram atendidas na Clínica Vitória MedifetUS, centro terciário de medicina fetal. Todos exames foram realizados pelos autores (CGVM e LCF) e os dados obtidos fizeram parte da planilha de estudo da tese de doutoramento do primeiro autor ${ }^{11}$. O estudo foi aprovado pela Comissão de Normas Éticas e Regulamentares da UNIFESP. O caráter do estudo foi explicado por meio de carta informativa e verbalmente a todas as pacientes em entrevista inicial, a fim de que pudessem expressar, por escrito, seu consentimento para a realização do exame. Todos os exames foram fotografados e/ou gravados em vídeo para posterior estudo das imagens obtidas.

Foram utilizadas as vias vaginal e abdominal a fim de se obter a melhor imagem, sendo que na maioria das vezes obteve-se sucesso com a via vaginal. $\mathrm{Na}$ realização dos exames foi utilizado aparelho marca Toshiba, modelo SSH-140 A, com transdutores de 6,0 e 3,5 $\mathrm{MHz}$.

Em 120 casos realizou-se estudo citogenético no material obtido por meio da biopsia de vilosidade coriônica e em 4 por meio da amniocentese. $O$ resultado teve como base o fenótipo do recém-nascido em 1028 casos. Portanto, o padrão ouro utilizado foi o cariótipo e/ou o fenótipo do recém-nascido.

Todos os fetos foram submetidos à ultra-sonografia de rotina e à medida da TN entre a $10^{\mathrm{a}} \mathrm{e}$ $14^{\mathrm{a}}$ semanas. Em todos os casos a avaliação do risco para cromossomopatia foi realizada mediante a combinação da idade materna e da medida da $\mathrm{TN}^{3}$. Após o aconselhamento genético, coube ao casal a decisão quanto à realização do procedimento invasivo para confirmação do diagnóstico.

A medida da TN foi aferida por meio da imagem no plano sagital do feto, o mesmo utilizado para a avaliação do comprimento cabeça-nádegas (CCN), com a coluna posteriormente localizada. Mediu-se o espaço hipoecogênico entre a pele e o tecido celular subcutâneo que recobre a coluna cervical, previamente definido ${ }^{1}$.

Os resultados foram formulados em termos de suspeição ou não de anomalias cromossômicas. Para suspeição das aneuploidias adotamos o percentil maior que 95 , além dos pontos de corte de 2,5 e $3 \mathrm{~mm}$, e ainda, os riscos corrigidos de $1 / 300,1 / 250$ e $1 / 50$. O percentil 95 teve origem dos casos normais do estudo.

Para determinar o significado das diferenças entre as médias das variáveis analisadas no grupo com resultado normal e no grupo com anomalia cromossômica, foi utilizado o teste paramétrico $t$ de Student.

Para analisar as características do teste (medida da TN) calcularam-se: sensibilidade (S), especificidade (E), valor preditivo positivo (VPP), valor preditivo negativo (VPN), probabilidade do falso-positivo (PFP = 1 - VPP), probabilidade do falso negativo $(\mathrm{PFN}=1-\mathrm{VPN})$ e razão de probabilidade positiva ( $R P+$ ) e negativa (RP-) ou razão de verossimilhança ou likelihood ratio, que expressa a pro- 
babilidade de testes positivos nas cromossomopatias dividido pelos positivos no grupo normal, isto para $\mathrm{RP}+$. Calculou-se, também, o que chamamos de taxa de falso-positivo com base na fórmula: $(\mathrm{TFP}=1-\mathrm{E})$. Inicialmente foi considerada a prevalência $(\mathrm{P})$ da população estudada como sendo a prevalência verdadeira. Posteriormente foram calculados VPP, VPN, PFP, PFN e RP, considerando-se outras prevalências para trissomia do $21(1 / 1000$, $1 / 700,1 / 300,1 / 100$ e 1/50, equivalente à idade materna de $29,31,35,40$ e 42 anos, respectivamente) $)^{3}$. Esse cálculo foi realizado por meio da fórmula: $\mathrm{VPP}=\mathrm{S} \times \mathrm{P} /(\mathrm{S} \times \mathrm{P})+(1-\mathrm{E}) \times(1-\mathrm{P}) \mathrm{e}$ $\mathrm{VPN}=(1-\mathrm{P}) \times \mathrm{E} / \mathrm{P}(1-\mathrm{S})+(1-\mathrm{P}) \mathrm{E}$.

\section{Resultados}

Após analisados os dados e excluídos os casos conforme critérios definidos previamente, restaram 1152 fetos nos quais a medida da TN foi adequada. Nenhum caso foi excluído por não ter sido possivel realizar uma boa avaliação. Em 651 $(56,5 \%)$ oportunidades, utilizamos a via vaginal; em 442 oportunidades $(38,3 \%)$ foi utilizada a via abdominal e em $59(5,1 \%)$ usamos as duas vias. Nos primeiros 500 exames acrescentaram-se em média 5 minutos ao tempo dispensado para se conseguir mensuração do CCN. Posteriormente esse tempo foi reduzido para 1 minuto em média.

A distribuição conforme a idade gestacional foi de 115 casos $(10,9 \%)$ com 10 semanas de gestação, 334 casos $(28,9 \%)$ com 11 semanas, 415 casos $(36 \%)$ com 12 semanas, 276 casos $(23,9 \%)$ com 13 semanas e $12(1 \%)$ com 14 semanas de gestação.

Entre as características da população estudada, a idade materna variou de 14 a 49 anos, com média de 30,2 anos. Do total de 1152 pacientes, $225(22,2 \%)$ encontravam-se com 35 ou mais anos de idade. Nessa faixa etária, $56(22 \%)$ submeteram-se aos procedimentos invasivos. Em relação às gestantes com idade inferior a 35 anos, 68 de $927(7,3 \%)$ realizaram o estudo citogenético. As 68 pacientes com idade inferior a 35 anos submeteram-se ao procedimento por outras razões: antecedente ou história familiar de cromossomopatia, ansiedade e TN aumentada.

A prevalência de anomalias cromossômicas na população estudada foi de 1,99\%. Dentre os 23 casos de anomalias cromossômicas, o estudo citogenético concluiu pela síndrome de Down em 14 casos. Os outros diagnósticos foram: trissomias 9, 18, 22, triploidia, triplo X, síndrome de Klinefelter e sindrome de Turner (Tabela 1).
Tabela 1 - Idade materna, idade gestacional (IG) e medida da translucência nucal (TN) nos 23 casos com alterações cromossômicas.

\begin{tabular}{|c|c|c|c|c|}
\hline Número & Idade materna & IG (semanas) & $\mathrm{TN}(\mathrm{mm})$ & Cariótipo \\
\hline 1 & 29 & 11 & 2,5 & Trissomia 22 \\
\hline 2 & 41 & 12 & 6,0 & Trissomia 21 \\
\hline 3 & 41 & 13 & 5,9 & Trissomia 21 \\
\hline 4 & 32 & 11 & 1,5 & Trissomia 21 \\
\hline 5 & 40 & 11 & 1,7 & Trissomia 21 \\
\hline 6 & 25 & 13 & 4,4 & Trissomia 21 \\
\hline 7 & 17 & 13 & 4,9 & Turner \\
\hline 8 & 22 & 12 & 1,6 & Trissomia 18 \\
\hline 9 & 31 & 11 & 4,5 & Trissomia 21 \\
\hline 10 & 28 & 12 & 4,8 & Trissomia 21 \\
\hline 11 & 40 & 13 & 1,6 & Klinefelter \\
\hline 12 & 31 & 10 & 1,4 & Trissomia 21 \\
\hline 13 & 41 & 12 & 6,5 & Trissomia 21 \\
\hline 14 & 33 & 10 & 3,5 & Triplo X \\
\hline 15 & 29 & 12 & 7,1 & Trissomia 18 \\
\hline 16 & 38 & 12 & 4,0 & Trissomia 21 \\
\hline 17 & 40 & 12 & 2,3 & Trissomia 21 \\
\hline 18 & 29 & 14 & 12,7 & Turner \\
\hline 19 & 42 & 12 & 3,6 & Trissomia 21 \\
\hline 20 & 23 & 13 & 4,1 & Triploidia \\
\hline 21 & 32 & 12 & 4,2 & Trissomia 21 \\
\hline 22 & 30 & 12 & 9,1 & Trissomia 9 \\
\hline 23 & 32 & 10 & 1,7 & Trissomia 21 \\
\hline
\end{tabular}

A Tabela 2 mostra que o CCN médio foi 59,2 $\mathrm{mm}$ (variando de 38 a $84 \mathrm{~mm}$ ). A idade gestacional média no momento da avaliação foi 12,1 semanas (variando de 10 semanas e 3 dias a 14 semanas e 2 dias). Nos casos com anomalias cromossômicas, a medida da TN variou de 1,4 a 12,7 $\mathrm{mm}$ (média de 4,3 $\mathrm{mm}$ ); já no grupo de fetos normais, a TN variou de 0,6 a $6,6 \mathrm{~mm}$ (média de $1,6 \mathrm{~mm})(\mathrm{p}<0,001)$.

Tabela 2 - Média dos valores para a idade materna, translucência nucal (TN) e comprimento cabeça/nádegas (CCN) no grupo de fetos normais e no grupo de fetos portadores de anomalias cromossômicas.

\begin{tabular}{lccccc}
\hline Variável & \multicolumn{2}{c}{ Normal } & \multicolumn{3}{c}{ Cromossomopatia } \\
& \multicolumn{2}{c}{$(\mathbf{n = 1 1 2 9 )})$} & \multicolumn{2}{c}{$(\mathbf{n = 2 3 )}$} & \\
& Média & DP & Média & DP & valor de $\mathbf{p}$ \\
\hline Idade materna & 30,2 & 5,5 & 32,4 & 7,0 & $=0,057$ \\
TN & 1,6 & 0,6 & 4,3 & 2,8 & $<0,001$ \\
CCN & 59,2 & 11,7 & 60,2 & 12,7 & NS \\
\hline DP = desvio padrão; NS = não significante. & & &
\end{tabular}

$\mathrm{DP}=$ desvio padrão; $\mathrm{NS}=$ não significante. 
A Figura 1 mostra o gráfico da distribuição dos casos normais e anormais em relação ao CCN e à medida da TN (percentis 5 e 95).

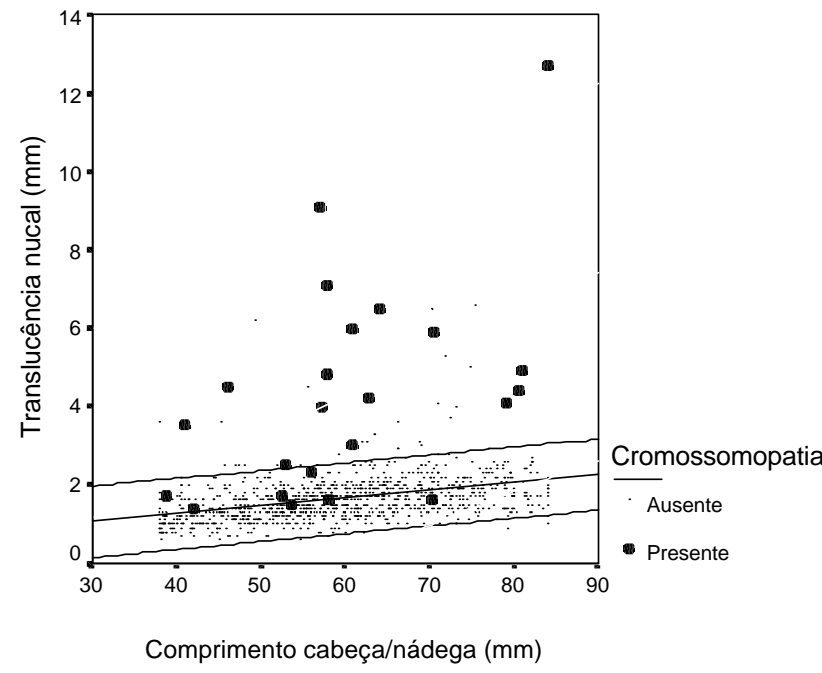

Figura 1 - Relação entre a medida da translucência nucal e o comprimento cabeça/ nádegas, em fetos normais $(n=1129)$ e com anomalias cromossômicas $(n=23)$, projetados num gráfico com distribuição dos valores normais da translucência nucal para 0 comprimento cabeça/nádegas $\left(95^{\circ}\right.$ e $5^{\circ}$ percentil).

Verificou-se que a idade materna de 35 anos isoladamente apresenta uma baixa sensibilidade diagnóstica $(34,8 \%)$ associada à alta taxa de resultados falso-positivos $(22,0 \%)$.

Quanto à diferença no valor de corte para $\mathrm{TN}$, verificamos que a redução de $3,0 \mathrm{~mm}$ para $2,5 \mathrm{~mm}$ apresentou discreto aumento na sensibilidade $(65,2 \%$ para $69,6 \%)$, às custas de elevação das taxas de resultados falso-positivos $(1,9 \%$ para $6,9 \%$ ), e da probabilidade de falso-positivos $(59,5 \%$ para $82 \%)$.

Com relação aos limites percentílicos, observamos que o limite do $95^{\circ}$ percentil garantiu a mesma sensibilidade observada no valor de corte de $2,5 \mathrm{~mm}(69,5 \%)$ associada à discreta elevação nas taxas de resultados falso-positivos $(1,9 \%$ para $3,7 \%$ ), o que não foi observado com o percentil 97,5 .

Ao analisarmos as razões de probabilidades de um resultado alterado associadas à presença da anomalia cromossômica fetal, verificamos que os melhores parâmetros são: valor de corte de 3,0 $\mathrm{mm}(\mathrm{RP}+=33,5), 97,5^{\circ}$ percentil $(\mathrm{RP}+=23,8)$ e $95^{\circ}$ percentil $(\mathrm{RP}+=19,1)$.

Com relação ao risco populacional ideal para a indicação dos procedimentos invasivos verificamos que a elevação do risco de $1 / 300$ para $1 / 250$ apenas reduziu a sensibilidade do método, sem haver melhora em nenhum dos outros parâmetros, e que apenas elevações na ordem de 1/50 foram capazes de reduzir a probabilidade de falsopositivos $(9,7$ para 2,5$)$ e elevar as razões de probabilidades positivas $(8,0$ para 26,3$)$.
Na Tabela 3 observa-se que o cariótipo concluiu por cromossomopatia em 16 dos 57 fetos que apresentaram TN com medida acima do percentil 95. Dos 1095 fetos que exibiram TN menor que o $95^{\circ}$ percentil, apenas 7 apresentaram cariótipo anormal, resultando em uma sensibilidade de $69,5 \%$, especificidade de $96,3 \%$, valor preditivo positivo de $28,0 \%$, valor preditivo negativo de $99,3 \%$ e probabilidade de falso-positivo de 72,0\%, com base na fórmula 1 - VPP, e de $3,7 \%$, quando usada a fórmula 1 - $\mathrm{E}$. A razão de probabilidade positiva foi de 19,1 .

Tabela 3 - Medida da translucência nucal em fetos normais e com anomalias cromossômicas entre 10 e 14 semanas de gestação. 0 asterisco significa presença de 1 caso de hipoplasia do coração esquerdo (malformação cardíaca principal).

\begin{tabular}{|c|c|c|}
\hline $\begin{array}{c}\text { Translucência } \\
\text { nucal }\end{array}$ & $\begin{array}{l}\text { Fetos normais } \\
\qquad(\mathrm{n}=1129)\end{array}$ & $\begin{array}{l}\text { Anomalias cromossômicas } \\
\qquad(\mathrm{n}=23)\end{array}$ \\
\hline $\begin{array}{c}<\text { percentil } 95 \\
(n=1095)\end{array}$ & 1088 & $\begin{array}{l}\text { Trissomia } 21=05 \\
\text { Trissomia } 18=01 \\
\text { Klinefelter }=01\end{array}$ \\
\hline $\begin{array}{l}>\text { percentil } 95 \\
\quad(n=57)\end{array}$ & $41^{*}$ & $\begin{array}{l}\text { Trissomia } 21=09 \\
\text { Turner }=02 \\
\text { Trissomia } 22=01 \\
\text { Trissomia } 18=01 \\
\text { Trissomia } 9=01 \\
\text { Triploidia = } 01 \\
\text { Triplo } X=01\end{array}$ \\
\hline
\end{tabular}

\section{Discussão}

A medida da TN, considerada excelente meio de rastreamento da síndrome de Down, deve ser adotada como rotina em todos os centros diag-nósticos, após treinamento específico de cada examinador. A Fetal Medicine Foundation do Brasil ministra cursos tutoriais de excelência, mantendo auditoria periódica dos diversos centros cadastrados, garantindo a qualidade dos exames de rastreamento.

Para melhor eficácia do rastreamento das anomalias cromossômicas com base na medida da TN, recentemente, a Fetal Medicine Foundation ${ }^{15}$ fez as seguintes recomendações: o feto deve ter no mínimo $45 \mathrm{~mm}$ de CCN e no máximo $84 \mathrm{~mm}$; o exame pode ser feito por via abdominal ou vaginal, mas a via vaginal permite maior reprodutibilidade do método. Deve-se realizar um bom corte sagital do feto (o mesmo para medir o $\mathrm{CCN}$ ), e o feto deve ocupar no mínimo três quartos da imagem amplificada. É importante discriminar a membrana amniótica da pele fetal, devendo-se medir a espessura máxima do espaço hipoecogênico, en- 
tre a pele e o tecido celular subcutâneo que recobre a coluna cervical. Para isso o feto deverá estar em posição neutra (ausência de hiperextensão ou flexão do pescoço) e os "calipers" deverão ter a forma de "+" de modo que a linha horizontal do "caliper" fique na linha que delimita a TN. Caso haja circular cervical do cordão (5-10\% dos casos), as medidas da TN acima e abaixo do cordão são diferentes; para cálculo de risco, recomenda-se adotar a menor medida.

A experiência aqui referida mostrou que 16 dos 23 fetos com aneuploidias exibiram medida da TN maior que o percentil 95, taxa de detecção de $69,5 \%$. Esse critério (percentil 95) foi o que apresentou melhor acurácia na discriminação entre fetos normais e anormais.

Embora haja alguns resultados contraditórios na literatura, a medida da TN é considerada excelente meio de rastreamento de cromossomopatias, particularmente pelo grupo do Kings College $^{3}$. Relatamos resultados preliminares que nos permitem vislumbrar a utilização desse novo método no rastreamento de anomalias cromossômicas. Além disso, o aumento da TN no primeiro trimestre da gestação poderá ser um sinal de alerta para a investigação de defeitos cardíacos, especialmente naqueles casos que apresentam cariótipo normal.

Os dados do presente estudo confirmam os resultados da literatura, os quais evidenciam que o aumento da TN não só aumenta o risco para anomalia cromossômica, como para cardiopatia congênita $^{1-3}$.

$\mathrm{Na}$ intersecção dos estudos realizados, a pretexto de referência às medidas seriadas da TN e da época mais conveniente ao rastreamento das trissomias autossômicas, Borrell et al. ${ }^{16}$ concluem serem a $12^{a}$ e a $13^{a}$ semanas as datas apropriadas em relação à síndrome de Down e à trissomia do 18 . Ao reexame $(17$ fetos com trissomia 18), no transcurso da $10^{a}$ para a $20^{a}$ semana (média das idades gestacionais na primeira e segunda aferição, respectivamente 13,5 e 16,5 semanas), o espessamento nucal (translucidez) mostrou valores absolutos crescentes. Contudo, não se legitima a pequena ascendência, visto que o correspondente desvio padrão (DP) médio de 0,2 é bastante inferior ao que se convencionou ado$\operatorname{tar}(\mathrm{DP}>2,5$ ) em conformidade com a idade fetal, como aumento genuíno na amplitude da $\mathrm{TN}^{17}$.

No nosso meio, Brizot et al. ${ }^{18}$ analisaram a validade da medida ultra-sonográfica da TN no rastreamento de anomalias cromossômicas entre 10 e 14 semanas de gestação, estudando 2996 fetos consecutivamente. Foram diagnosticados 22 casos de anomalias cromossômicas, incluindo 10 trissomias do cromossoma 21. Com base na esti- mativa de risco da idade materna associada à medida da TN, o risco de 1/300 foi capaz de detectar 9 dos 10 casos de sindrome de Down (90\%) e 9 dos 12 casos referentes às outras anomalias cromossômicas (75\%), ao passo que a medida da TN acima do percentil 95 foi capaz de detectar $70 \%$ das trissomias do 21. Os autores concluíram que a performace do teste na população brasileira é semelhante à da população britânica.

Os nossos resultados foram bastante semelhantes ao da literatura, com taxa de detecção de aproximadamente $70 \%$ e com taxa de falso-positivo de menos de 5\%. Este baixo percentual de resultado falso-positivo que é enfatizado por vários autores foi obtido por meio da fórmula 1 - E, quando deveria ser utilizada 1 - VPP. Essa diferença é importante porque no primeiro caso pode haver erros de interpretação, visto que esse cálculo não leva em consideração a prevalência na população estudada. Na experiência ora relatada a probabilidade de falso-positivo subiu de 3,6 para 71,9 , quando foi adotada a fórmula 1 - VPP. Acreditamos ser a probabilidade de falso-positivo um valor de maior utilidade na prática clínica durante o aconselhamento do risco para aneuploidias.

Outro critério avaliado no desempenho da medida da TN no rastreamento de anomalias cromossômicas foi a razão de probabilidade positiva $(\mathrm{RP}+)$, que foi de 19,1 ao empregar o $95^{\circ}$ percentil. A RP ou razão de verossimilhança ou likelihood ratio é definida como a probabilidade de resultado alterado em pessoas com a doença, dividida pela probabilidade do mesmo resultado em pessoas sem a doença. No estudo em apreço a RP expressou que a medida da TN alterada é 19 vezes mais provável de ser positiva nos casos de anomalias cromossômicas do que nos fetos normais.

Um teste de rastreio deve ser aplicado a uma população aparentemente normal com o intuito de identificar indivíduos com alta probabilidade de terem uma determinada doença. Um teste de rastreio positivo acaba por originar, na maior parte dos casos, a realização de um teste diagnóstico. Um bom teste de rastreamento, portanto, deve ter alta sensibilidade e alta especificidade, para reduzir o número de resultados falso-positivos que necessitem posterior investigação. Devido à baixa prevalência das doenças em geral, o valor preditivo da maior parte dos testes de rastreamento é baixo, mesmo para testes de alta especificidade. Assim, a eficácia de um teste de rastreio depende basicamente da prevalência da doença na população: quanto mais baixa for a prevalência de aneuploidia na população rastreada, mais baixo é o valor preditivo positivo e mais elevada é a taxa de falso-positivos. Para que um teste de rastreio tenha significado clínico, torna-se necessário que 
a doença seja suficientemente prevalente na população estudada. A doença que se pretende detectar deve ser responsável por morbidade e/ou mortalidade significativa e, uma vez detectada, devem existir possibilidades de diagnóstico e de conduta subseqüentes. O teste deve ainda ser aceito pela população, ser reprodutivel e válido (deve conseguir medir o que se pretende medir) e apresentar uma relação custo/benefício razoável. Deste modo, faz parte da natureza essencial de um teste de rastreio um compromisso entre a sensibilidade do teste e o seu valor preditivo positivo, de forma a selecionar um padrão para que o resultado do rastreio possa ser considerado positivo ${ }^{19}$.

No estudo em apreço, a prevalência da sindrome de Down foi de 1/ 82 (14 casos em 1152), ou seja, bastante alta. Isto significa que os resultados, sobretudo os valores preditivos, não são válidos para a população geral. Outro aspecto digno de nota referente à pesquisa em tela é que o padrão ouro foi o fenótipo do recém-nascido na maioria dos casos normais. Isso significa que pode haver, teoricamente, casos de cromossomopatias entre os casos considerados normais pelo fenótipo. Isso aconteceria, eventualmente, em aneuploidias com poucas expressões fenotípicas, particularmente nas anomalias cromossômicas ligadas ao sexo.

A TN é um exemplo de um teste de rastreio eficaz, já que é simples, pouco dispendioso, reprodutivel, seguro, sensivel e tem boa aceitabilidade por parte das pacientes e dos clínicos. Além disso, a medida da TN detecta uma entidade prevalente na população que, depois da suspeição, é passível de ser diagnosticada ${ }^{19}$. Entretanto a taxa de falso-positivo é de $5 \%$, o que significa ser desnecessário, em população de 20.000 gestantes, o procedimento invasor em 1000 , o que ocasionaria abortamento em cerca de 10 (1\%), sem contar o ônus econômico e emocional ${ }^{11}$. A taxa de falso-positivo de 5\% é considerada aceitável quando se usa a fórmula 1 - VPP; tal não acontece na maioria dos estudos com menor expressão, que utilizam para o cálculo de probabilidade de falsopositivo a fórmula 1 - E.

São promissores os resultados observados com a dopplervelocimetria do duto venoso associada à aferição da TN na detecção destas síndromes no primeiro trimestre da gestação ${ }^{11}$. A alteração da onda de velocidade do fluxo no duto venoso, notadamente durante a contração atrial (DV zero/ reverso), no primeiro trimestre da gestação, estimula a investigação seqüencial dos defeitos cromossômicos. Provavelmente a realização da dopplervelocimetria do duto venoso associada à medida da TN possa subtrair a exigência do estudo citogenético em alguns $\operatorname{casos}^{11,20,21}$. Murta ${ }^{11}$ relata resultados correspondentes a 606 fetos e mostra que, por intermédio do DV zero/reverso, se identificaram $91,3 \%$ das trissomias do 21 , com especificidade de $96,1 \%$, valor preditivo positivo de $72,4 \%$ e valor preditivo negativo de $99,6 \%$. A baixa incidência de falso-positivo de 1,4\% (oito fetos), bem como as porcentagens que denotam a eficácia conferem supremacia relativa ao procedimento germinante. Entende-se que a combinação da medida da TN e a velocimetria no duto venoso restringem a indicação de procedimento invasor a menos de $0,5 \%{ }^{11}$.

Estando a dopplervelocimetria do duto venoso no início da utilização na prática obstétrica, não vale a pena comparar com a medida da TN, e sim, sugerir que com a associação de ambas, provavelmente em série, poderá haver tendência de diminuição da probabilidade de resultado falso-positivo e de maximização da probabilidade do valor preditivo positivo.

Do exposto, depreende-se que basta a análise quantitativa da medida da TN para categorizar o risco das anomalias cromossômicas no primeiro trimestre. Portanto, ainda que seja necessário treinamento e habilidade do operador, trata-se de método factível e de aplicabilidade clínica.

\section{ABSTRACT}

Purpose: to study the value of nuchal translucency (NT) measurement in the screening for chromosomal abnormalities at 10-14 weeks of gestation.

Methods: a total of 1152 fetuses were studied consecutively. In 124 cases a cytogenetic study was performed on material obtained from a biopsy of the chorionic villus, and in 1028 cases the result was based on the postnatal phenotype. In addition to the routine ultrasonographic examination, all fetuses were submitted to measurement of the NT thickness. For statistical analysis Student's test and ANOVA were used. Sensitivity, specificity, positive and negative predictive values, false-positive rate and likelihood ratio were calculated.

Results: twenty-three cases of chromosomal abnormalities occurred. Of these abnormal cases, NT measurement was above the 95 th percentile in 16 (sensitivity of $69.5 \%$ ). In the group of normal fetuses (1129 cases), NT measurement was above the 95th percentile in 41 (specificity of $96.3 \%$, positive and negative predictive values of $28.0 \%$ and $99.3 \%$, respectively, false-positive rate of $3.7 \%$ and likelihood ratio of 19.1).

Conclusion: our results suggest that the presence of chromosomal abnormalities may be strongly suspected when there is an increased NT thickness. One can infer that the quantitative NT analysis is sufficient to classify the risk of chromosomal anomalies in the first trimester of the pregnancy. 
Although the ultrasound operator's training and skill is still necessary, it is a method of clinical applicability.

KEY WORDS: Nuchal translucency. Chromosomal abnormalities. Prenatal diagnosis.

\section{Agradecimentos}

Agradecemos o suporte da Fetal Medicine Foundation do Brasil, notadamente à Dra. Maria de Lourdes Brizot.

\section{Referências}

1. Nicolaides KH, Azar G, Snijders RJ, Gosden CM. Fetal nuchal oedema: associated malformations and chromosomal defects. Fetal Diagn Ther 1992; $7: 123-31$.

2. Pandya PP, Snijders RJ, Johnson SP, De Lourdes Brizot M, Nicolaides KH. Screening for fetal trisomies by maternal age and fetal nuchal translucency thickness at 10 to 14 weeks of gestation. Br J Obstet Gynaecol 1995; 102:957-62.

3. Snijders RJ, Noble P, Seberi N, Souka A, Nicolaides KH. UK multicentre project on assessment of risk of trisomy 21 by maternal age and fetal nuchal translucency thickness at 10-14 weeks of gestation. Lancet 1998; 352:343-6.

4. Spencer K, Souter V, Tul N, Snijders R, Nicolaiders $\mathrm{KH}$. A screening program for trisomy 21 at 10-14 weeks using fetal nuchal translucency, maternal serum free beta-human chorionic gonadotropin and pregnancy-associated plasma protein-A. Ultrasound Obstet Gynecol 1999; 13:231-7.

5. Pandya PP, Goldberg H, Walton B, et al. The implementation of the first trimester scanning at 10-13 weeks' gestation and the measurement of fetal nuchal translucency thickness in two maternity units. Ultrasound Obstet Gynecol 1995; 5:20-5.

6. Kornman LH, Morssink LP, Beekhuis JR, De Wolf BT, Heringa MP, Mantingh A. Nuchal translucency cannot be used as a screening test for chromosomal abnormalities in the first trimester of pregnancy in a routine ultrasound practice. Prenat Diagn 1996; 16:797-805.

7. Taipale P, Hjilesmaa V, Salonen R, Ylöstalo P. Increased nuchal translucency as a marker for fetal chromosomal defects. N Engl J Med 1997; 337:1654-8.

8. Pajkrt E, van Lith JM, Mol BW, Bleker OP, Bilardo CM. Screening for Down's syndrome by fetal nuchal translucency measurement in a general obstetric population. Ultrasound Obstet Gynecol 1998; 12:163-9.
9. Murta CGV, Moron AF, Avila MAP. Translucência nucal: mecanismos fisiopatológicos. GO Atual 1999; 6:24-8.

10. Montenegro N, Matias A, Areias JC, Castedo S, Barros H. Increased fetal nuchal translucency: possible involvement of early cardiac failure. Ultrasound Obstet Gynecol 1997; 10:265-8.

11. Murta CGV. Dopplerfluxometria aplicada ao duto venoso no primeiro trimestre: ênfase na detecção das aneuploidias [tese]. São Paulo: Universidade Federal de São Paulo; 2001.

12. Hyett JA, Brizot ML, von Kaisenberg CS, McKie AT, Farzaneh F, Nicolaides KH. Cardiac gene expression of atrial natriuretic peptide and brain natriuretic peptide in trisomic fetuses. Obstet Gynecol 1996; 87:506-10.

13. Hyett JA, Moscoso G, Nicolaides KH. First-trimester nuchal translucency and cardiac septal defects in fetuses with trisomy 21. Am J Obstet Gynecol 1995; 172:1411-3.

14. Hyett JA, Moscoso G, Nicolaides KH. Increased nuchal translucency in trisomy 21 fetuses: relationship to narrowing of the aortic isthmus. Hum Reprod 1995; 10:3049-51.

15.Nicolaides KH, Sebire NJ, Snijders RJ. The diagnosis of fetal abnormalities: the 11-14 week scan. $1^{\text {st }}$ ed. Casterton Hall: Parthenon; 1999; p. 15-6.

16.Borrell A, Farré MT, Echevarria M, Martinez JM, Costa D, Fortuny A. Nuchal thickness evolution in trisomy 18 fetuses. Ultrasound Obstet Gynecol 2000; 16:146-8.

17.Ávila MAP, Murta CGV, Moron AF. Duto venoso: curso primordial? Femina 2001; 29:9-13.

18. Brizot ML, Carvalho MH, Liao AW, Reis NS, Armbruster-Moraes E, Zugaib M. First-trimester screening for chromosomal abnormalities by fetal nuchal translucency in a Brazilian population. Ultrasound Obstet Gynecol 2001; 18:652-5.

19. Matias A. Retorno venoso na avaliação da função cardíaca fetal [tese]. Porto: Universidade do Porto; 2000.

20. Matias A, Gomes C, Flack N, Montenegro N, Nicolaides KH. Screening for chromosomal abnormalities at 10-14 weeks: the role of ductus venosus blood flow. Ultrasound Obstet Gynecol $1998 ; 12: 380-4$.

21.Murta CGV, Moron AF, Avila MAP. Dopplervelocimetria no rastreamento de aneuploidias no primeiro trimestre da gestação. Rev Bras Ginecol Obstet 2001; 23:291-8.

Recebido em: 24/07/01 Aceito com modificações em: 14/03/ 02 